\title{
Evaluation of the covariance matrix of neutronic cross sections with the Backward-Forward Monte Carlo method
}

\author{
E. Bauge, S. Hilaire, and P. Dossantos-Uzarralde \\ CEA DAM Île-de-France, Département de Physique Théorique et Appliquée, Service de Physique Nucléaire, BP. 12, \\ 91680 Bruyères-le-Châtel, France
}

\begin{abstract}
With the advent of modern nuclear reaction modeling codes, it has become possible to produce evaluated data from model calculations only. However, the process of estimating of the uncertainties associated with nuclear data evaluated by model calculations is not as well defined as the assessment of uncertainties resulting from an experimental-data-driven evaluation process. In this paper, we propose a method, based on the Monte Carlo sampling of the model parameter space, that allows for a quantitative estimation the covariance matrix of the model parameters, as well as that of the derived cross sections. Constrains from experimental data are included by building a probability density function of the model parameter space as a function of the generalized $\chi^{2}$ that estimates the calculationdata mismatch. The uncertainties of the model parameters are then propagated to the derived cross sections, whose distribution is analyzed in terms of a covariance matrix. As an example, this method is applied to the models used in the nuclear reaction code TALYS and a full covariance matrix for the evaluated cross sections of the $n+{ }^{89} \mathrm{Y}$ system is obtained.
\end{abstract}

\section{Introduction}

In recent years the common practice of nuclear data evaluation has started to shift from being driven by experimental data to being model-driven. That shift corresponds to the wide availability of nuclear model codes [1,2] and to the evolving nuclear data needs which extend farther away from the well measured nuclei of the stability line, thus forcing the evaluators to rely more on modeling.

Another evolution of nuclear data needs is the increasing focus on nuclear data covariances. Indeed, in order to guaranty the operating margins of their designs, the designers of new nuclear technologies (Generation IV reactors for example) need to assess the uncertainty of their simulations. To do so they have to propagate all the uncertainties associated with all the constants used in simulations towards the key working parameters of their designs. Underestimation of the final operating margins might result in a non working or unsafe design, while overestimation of these margins leads to "overengineered", sub-optimal, expensive designs. Thus the accurate estimation of the uncertainties associated with constants (nuclear cross sections for example) used in simulations is extremely important for application designers.

The current interest in covariances is manifest since at least two working groups of the Working Party on International Nuclear Data Evaluation Cooperation (WPEC) [3] are devoted to this topic (subgroups 24 and 26). Moreover, the COVAR mailing list [4] hosted by OECD Nuclear Energy Agency, features regular and high quality discussion on this topic by contributors from around the world.

In the continuum region, the methods used to compute covariance matrices in an experimental data driven evaluation are well known and are based on Bayesian inference. Such methods rely on the availability of relevant experimental data to update the prior knowledge of the evaluated cross section with experimental evidence. For example, the new covariance information of the ENDF/B.VII evaluated library was obtained using the KALMAN-EMPIRE [5] methodology based on Bayesian inference.

In this work we propose an alternative way of generating the covariance matrix associated with evaluated data: a method that is more relevant in the framework of model driven evaluation. This method, named Backward-Forward Monte Carlo (BFMC) relies on the Monte Carlo sampling of the model parameter space using experimental data to steer the sampling to the region where the agreement between calculation and the experimental data is optimal. We will first apply this method to the semi-microscopic optical model potential [6], and then extend the calculation to the models used in TALYS [1] with the example of the $n+{ }^{89} \mathrm{Y}$ system.

\section{Backward-Forward Monte Carlo}

The basis of the BFMC method relies on the assumption that the only source of the uncertainty of the calculated cross section is the imperfect knowledge of the model parameters. The two phases (Backward and Forward) correspond to the determination of the model parameter covariance matrix, and to the propagation of this matrix towards that of cross sections by Monte-Caro sampling ${ }^{1}$, respectively.

\subsection{Forward Monte Carlo}

In $[7,8]$, we used Monte Carlo sampling of optical model parameter space to propagate the assumed uncertainties of model parameters towards cross section uncertainties. This

1 In this paper, by sampling we mean a set of samples (or Monte Carlo "events") that represents a given probability distribution function. 


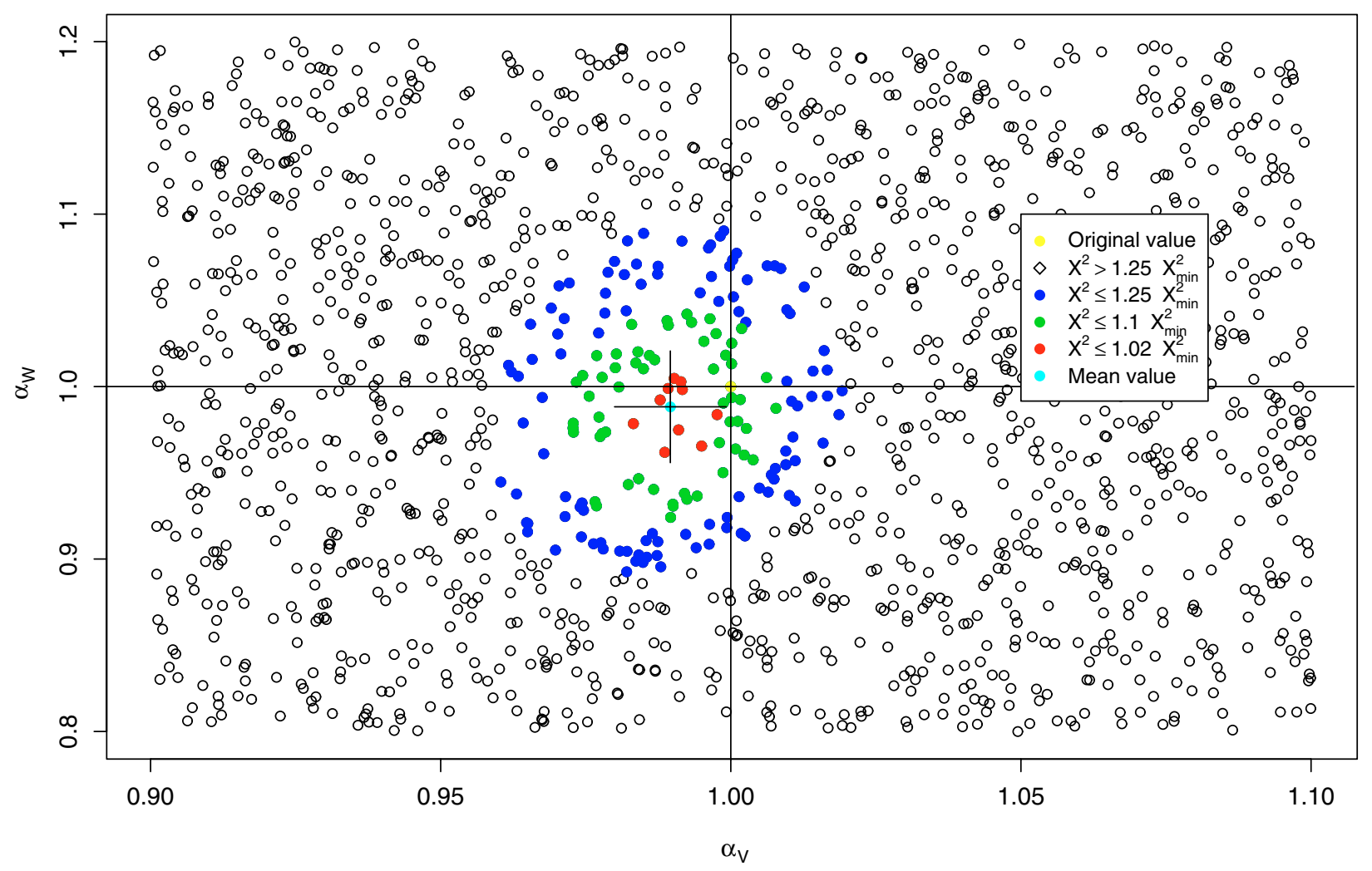

Fig. 1. Projection along the $\lambda_{V}, \lambda_{W}$ plane, of the $\chi^{2}$ distribution obtained by sampling (1500 events here for clarity) the four dimensional OMP parameter space. The axes are plotted as a function of the reduced parameters $\left(\alpha_{i}=\lambda_{i} / \lambda_{i 0}\right.$ where $\lambda_{i 0}$ is the optimal value given in [6]). Colors correspond to intervals of $\chi^{2}$ values.

work is the basis of the Forward Monte Carlo propagation of the model parameter covariance matrix. At the time the covariance matrix of the semi-microscopic optical model potential (SMOMP) [6] was assumed to be diagonal, and the variances were obtained from the sensitivity study of the response of the model to the independent variation of each parameter. Going beyond the above hypotheses, and assuming the known optimal value vector $P_{1}$ and covariance matrix $P_{2}$ of the $n$ model parameters $\left(p_{1}, \ldots, p_{n}\right)$, a probability distribution function (PDF) $P\left(p_{1}, \ldots, p_{n}\right)$ of the model parameters can be derived from the $n$-dimensional unit Gaussian distribution $G$ by

$$
P\left(p_{1}, \ldots, p_{n}\right)=\sqrt{P_{2}} G+P_{1}
$$

By construction, that $n$-dimensional Gaussian PDF has the same covariance matrix, $P_{2}$ and mean (optimal) value vector $P_{1}$ as the known optimal parameter distribution. By sampling $N$ times from $G$ and applying the transformation (1), a sampling of $P\left(p_{1}, \ldots, p_{n}\right):\left\{\left(p_{1}, \ldots, p_{n}\right)\right\}_{i=1, N}$ is obtained. That sampling of the model parameter space is then fed iteratively to the model to produce a sampling of the $m$ calculated observables (cross sections) $\left\{s_{1}, \ldots, s_{m}\right\}_{i=1, N}$ which is then analyzed in terms of mean value $S_{1}$ vector and covariance matrix $S_{2}$. That $S_{2}$ covariance matrix characterizes the dispersion of the calculated observables stemming from the response of the model to the PDF that represents the optimal parameter distribution. This covariance matrix represents the correlated uncertainty of the calculated observables.

\subsection{Backward Monte Carlo}

In the previous section, we started from a known parameter covariance matrix $P_{2}$ and optimal values vector $P_{1}$. While such information can be obtained by many least square fitting algorithms, we obtained it by a method based on the Monte Carlo sampling of the model parameter space. An uniform uncorrelated sampling (of size $N$ ) of the $n$-dimensional model parameter space $\left\{u_{1}, \ldots, u_{n}\right\}_{i=1, N}$ is fed into the model to produce a sampling of calculated cross section $\left\{s_{1}, \ldots, s_{m}\right\}_{i=1, N}$ corresponding to the parameter sampling. These cross sections are then compared with experimental data by calculating a generalized $^{2} \chi^{2}$ between data and each of the calculated cross section samples, producing the associated $\left\{\chi^{2}\right\}_{i=1, N}$ sampling. By weighting the uniform $\left\{u_{1}, \ldots, u_{n}\right\}_{i=1, N}$ model parameter sampling by a functional of the associated $\left\{\chi^{2}\right\}_{i=1, N}$ sampling, a sampling of a model parameter PDF which favors the values minimizing $\chi^{2}$, can be built. The chosen weighting function is

$$
w_{i}=C \exp \left[-\left(\frac{\chi_{i}^{2}}{\chi_{\text {min }}^{2}}\right)^{2}\right],
$$

where $\mathrm{C}$ is a normalization constant and $\chi_{\text {min }}^{2}$ is the minimal value of $\chi^{2}$ over the $\left\{u_{1}, \ldots, u_{n}\right\}_{i=1, N}$ parameter sampling. Note

\footnotetext{
${ }^{2} \chi^{2}$ calculated in a matrix form $\left(\chi^{2}=\sum_{i, j=1, m}\left(s_{i}-s_{i}^{e}\right) v_{i j}^{-1}\left(s_{j}-s_{j}^{e}\right)\right)$ that involves the full (non-diagonal) covariance matrix $\left(v_{i j}\right)$ of the experimental data that was inferred (and sometime "guesstimated") from the original experimental papers.
} 
Table 1. Distribution of the OMP experimental data sets by type of measurements with incident energies $10 \mathrm{MeV} \leq E \leq 65 \mathrm{MeV}$, and targets with masses $40 \leq A \leq 209$. DA denotes angular differential cross sections.

\begin{tabular}{ccc}
\hline Type of measurement & Nb of points & Nb of data sets \\
\hline (n, tot) & 1111 & 7 \\
(n, el) DA & 2132 & 67 \\
(p, reac) & 105 & 7 \\
(p, el) DA & 1883 & 52 \\
$(\mathrm{p}, \mathrm{n})^{I A S} \mathrm{DA}$ & 1095 & 36 \\
\hline
\end{tabular}

that $N$, the sample size, must be large enough for $\chi_{\min }^{2}$ to be a good representation of the minimum of the true (unsampled) $\chi^{2}$ distribution function. The $\left\{\pi_{i}\right\}_{i=1, N}=\left\{w_{i}\right\}\left\{u_{1}, \ldots, u_{n}\right\}_{i=1, N}$ sampling is a sampling of the PDF we have been looking for: the probability distribution function of the model parameters sets that produce observables which are consistent with the available experimental data. It is then easy to calculate the mean vector $\pi_{1}$ and covariance matrix $\pi_{2}$ associated with this model parameter PDF. $\pi_{1}$ and $\pi_{2}$ can then be introduced into equation (1) of section 2.1 for forward propagation of the covariance matrix of model parameters to that of the model calculated observables.

\section{Applications}

\subsection{Semi-microscopic optical model potential}

The semi-microscopic optical model potential (OMP) of [6] was used as a first test case for designing the BackwardForward Monte Carlo method. The nuclear central component of this OMP is built by folding the target radial density $\rho\left(r^{\prime}\right)$ with the OMP in nuclear matter $U_{N M}(\rho, E)$ using a Gaussian widening form factor of width $t$ :

$$
U(r, E)=(t \sqrt{\pi})^{-3} \int U_{N M}\left(\rho\left(r^{\prime}\right), E\right) e^{\left(-\left|r-r^{\prime}\right|^{2} / t_{r}^{2}\right)} d^{3} r^{\prime}
$$

The central OMP in nuclear matter is written as follows:

$$
\begin{aligned}
U_{N M}(\rho, E) & =\lambda_{V}(E)\left[V_{0}(\rho, \tilde{E}) \pm \lambda_{V 1}(E) \alpha V_{1}(\rho, \tilde{E})\right] \\
& +i \lambda_{W}(E)\left[W_{0}(\rho, \tilde{E}) \pm \lambda_{W 1}(E) \alpha W_{1}(\rho, \tilde{E})\right],
\end{aligned}
$$

with $V_{0}(\rho, E), V_{1}(\rho, E), W_{0}(\rho, E)$, and $W_{1}(\rho, E)$ the real isoscalar, real isovector, imaginary isoscalar and imaginary isovector components of the central OMP, respectively, which are calculated as shown in section II of ref. [9]. These components are re-normalized by the phenomenological normalization factors $\lambda_{V}, \lambda_{W}, \lambda_{V 1}$, and $\lambda_{W 1}$ adjusted to match a wide range of nucleon scattering and reaction observables for spherical and quasi-spherical targets. We will use these four normalization factors as the parameters which are the source of our uncertainties. In [6], diagonal uncertainties were given for these normalization factors, but no covariance matrix was obtained. The Backward Monte Carlo method was then used to obtain the full (non diagonal) covariance matrix of the four OMP parameters. The four OMP normalisations factors were
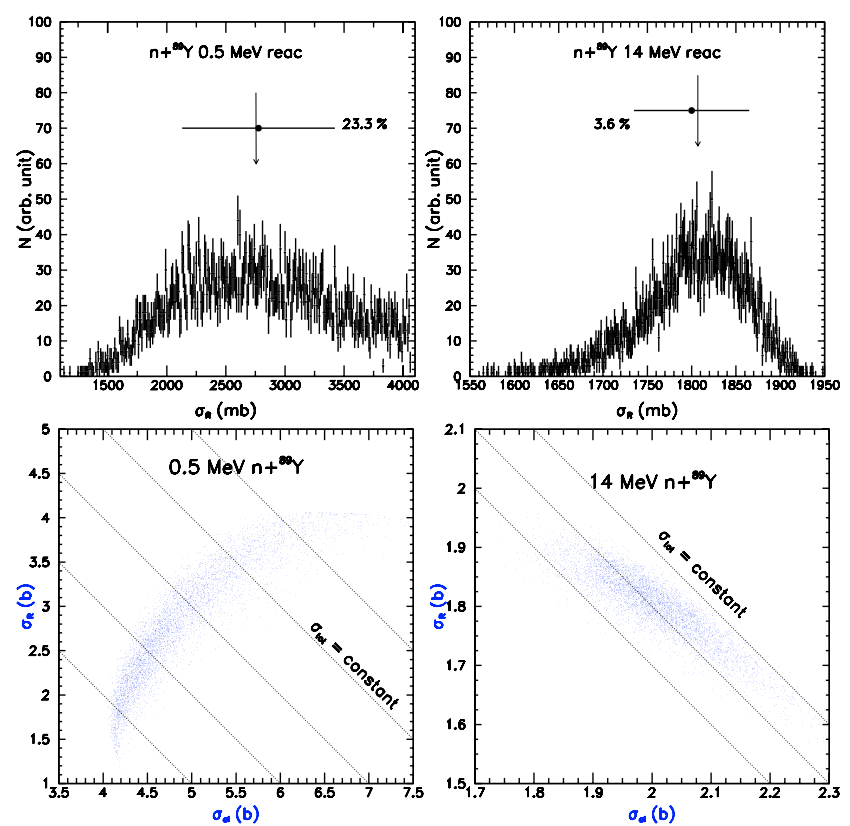

Fig. 2. Upper panels: Distribution of the reaction cross section for the system $\mathrm{n}+{ }^{89} \mathrm{Y}$ at 0.5 and $14 \mathrm{MeV}$ calculated using the BackwardForward Monte Carlo method. The vertical arrows show the cross sections values obtained with the OMP parameters of [6], and the horizontal error bars display the mean and standard deviation of the cross section distribution. Lower panel: correlated shape elastic, reaction and total cross sections for $n+{ }^{89} \mathrm{Y}$ at 0.5 and $14 \mathrm{MeV}$.

sampled independently with a uniform distribution of a $3 \sigma$ width ( $\sigma$ being the uncertainty quoted in [6]) 10000 times. The experimental data used to calculate the generalized $\chi^{2}$ values associated with each sampling of the parameter space is summarized in table 1 . The covariance matrix associated with each data set was estimated based on the information available in the original experimental papers. A two dimensional projection along the $\left(\lambda_{V}, \lambda_{W}\right)$ plane of the $\chi^{2}$ distribution is plotted in figure 1 . The first conclusion that can be drawn from this figure is that there is essentially no correlation between the two parameters $\lambda_{V}$ and $\lambda_{W}$ since the graph exhibits rotational symmetry around the minimal value. In order to be more quantitative, the covariance matrix $\pi_{2}$ and mean vector $\pi_{1}$ are extracted from the weighted (see eq. (2)) model parameter sample. The mean reduced parameter vector obtained is:

$$
\pi_{1}=\left(\begin{array}{l}
0.989 \\
0.988 \\
1.008 \\
0.966
\end{array}\right)
$$

which is very close to the unit vector, meaning that the original estimation [6] of the optimal parameter values was very close to the optimum. The standard deviation vector obtained from the diagonal part of the covariance matrix $\pi_{2}$ reads:

$$
\sqrt{\pi_{2 D}}=\left(\begin{array}{c}
0.0096 \\
0.0324 \\
0.176 \\
0.125
\end{array}\right) .
$$



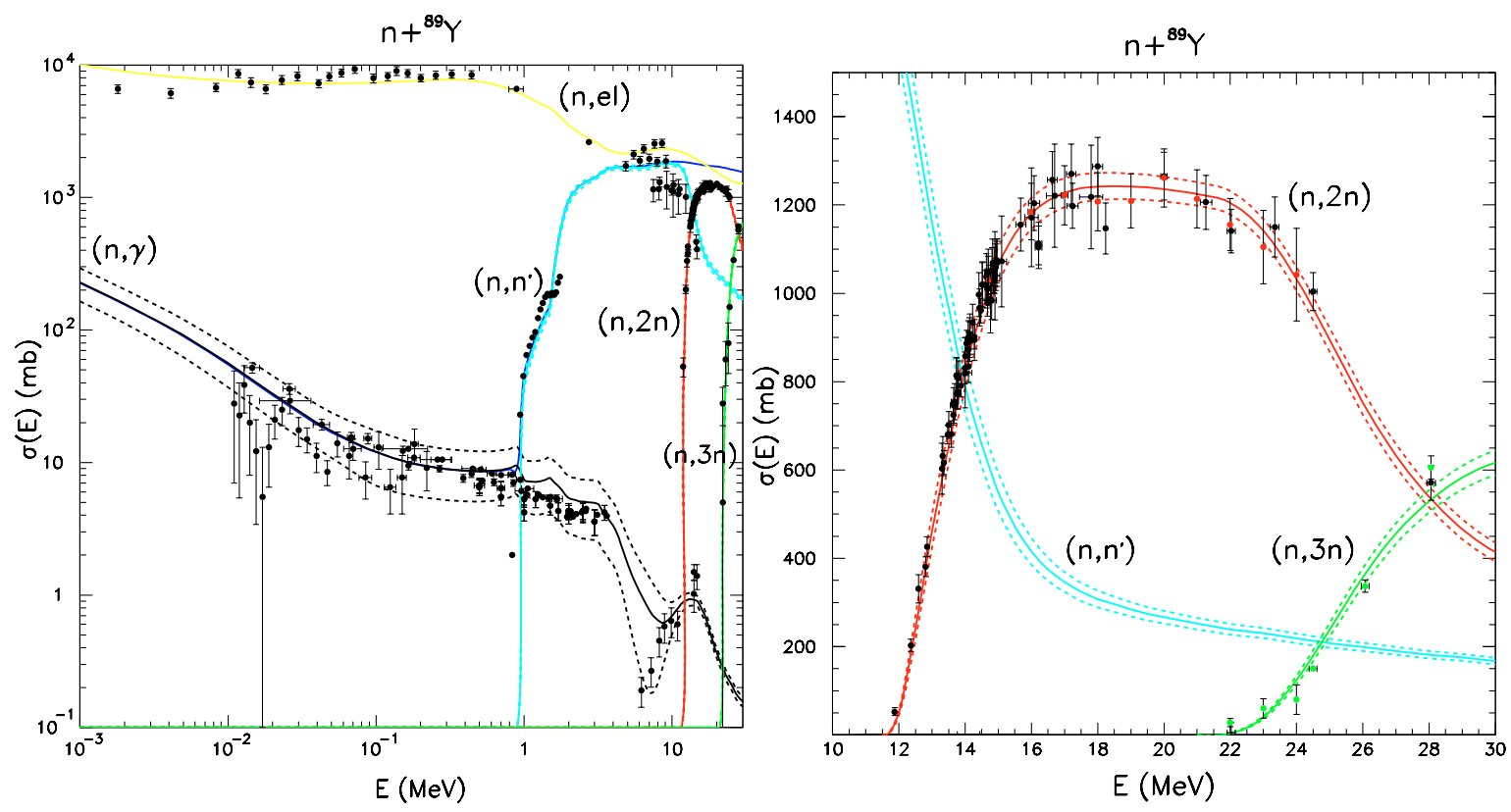

Fig. 3. Standard deviation band (dashed lines) for the $n+{ }^{89} Y$ reaction for the $(n, \gamma),\left(n, n^{\prime}\right),(n, 2 n)$ and $(n, 3 n)$ channels, compared with experimental data (symbols).

Table 2. Distribution of the experimental data sets by type of measurements for optimization of the six chosen TALYS model parameters with the BFMC method.

\begin{tabular}{ccc}
\hline Type of measurement & Nb of points & Nb of data sets \\
\hline$(\mathrm{n}, \gamma)$ & 109 & 12 \\
$(\mathrm{n}, \mathrm{el})$ & 26 & 4 \\
$\left(\mathrm{n}, \mathrm{n}^{\prime}\right)$ & 25 & 3 \\
$(\mathrm{n}, 2 \mathrm{n})$ & 80 & 12 \\
$(\mathrm{n}, 3 \mathrm{n})$ & 8 & 2 \\
\hline
\end{tabular}

Again these values are consistent (and compatible) with those given in [6]. The error bars plotted in figure 1 materialize these standard deviations in the $\left(\lambda_{V}, \lambda_{W}\right)$ plane. Finally, the correlation matrix calculated from the $\pi_{2}$ covariance matrix reads:

$\pi_{2 C}=\left(\begin{array}{llll}1.00 e+0 & 7.12 e-2 & -2.66 e-2 & 8.89 e-3 \\ 7.12 e-2 & 1.00 e+0 & -6.19 e-4 & -5.09 e-3 \\ -2.66 e-2 & -6.19 e-4 & 1.00 e+0 & 1.14 e-1 \\ 8.889 e-3 & -5.09 e-3 & 1.14 e-1 & 1.00 e+0\end{array}\right)$.

Examination of this correlation matrix shows that the correlations between the four OMP parameter are very weak, as could be "seen" from the $\left(\lambda_{V}, \lambda_{W}\right)$ projection of figure 1 . This very weak correlation is consistent with the hypothesis of independence of the four OMP parameters that was postulated in $[7,8]$.

The values of $\pi_{1}$ and $\pi_{2}$ can then be introduced in equation (1) to generate a sampling of the calculated cross sections $\left\{s_{1}, \ldots, s_{m}\right\}_{i=1, N}$ which can be analyzed in terms of mean value $S_{1}$ and covariance matrix $S_{2}$. The upper panels of figure 2 display the calculated reaction cross section distributions for $\mathrm{n}+{ }^{89} \mathrm{Y}$ at 0.5 and $14 \mathrm{MeV}$. These graphs show that the mean and standard deviation (horizontal error bars) of the BFMC calculated distributions are consistent with the cross section values calculated with the parameters of [6] (arrows). The standard deviation associated with the cross section distribution can be seen to be quite large at $0.5 \mathrm{MeV}$ (23\% of the mean value), while it stays fairly small $(3.5 \%)$ at $14 \mathrm{MeV}$. Similar calculations performed at other energies produce standard deviation that lie in between the above two extremes.

The lower panels of figure 2 show the shape elastic, reaction and total cross sections distributions for the same systems. These graphs illustrate the strong positive $(\mathrm{R}=0.91$ at $0.5 \mathrm{MeV})$ or negative $(\mathrm{R}=-0.87$ at $14 \mathrm{MeV})$ correlations generated by the response of the OMP to variations of its model parameters. When the correlation between shape elastic and reaction cross sections is positive, the total cross section $\left(\sigma_{T}=\sigma_{e l}+\sigma_{R}\right)$ observable provides a strong constraint to the OMP parameters values, and, conversely, when it is negative, a wide range of parameters values produce similar total cross section values. Thus, the energy ranges where $\sigma_{e l}-\sigma_{R}$ correlations are strongly positive define the ranges where the total cross section observable is the most sensitive to OMP parameters. Such information is valuable for everyone involved in OMP parameter search constrained by the total cross section.

\subsection{TALYS Calculation of $n+{ }^{89} Y$}

In order to perform an evaluation with TALYS, several parameters have to be adjusted in order to match the available experimental data. Besides the four OMP parameters whose covariance matrix and mean vector are already optimized vs. scattering observables (see section 3.1), six other model parameters were introduced in the BFMC process. Those are $\Gamma \gamma_{90}$ the $\gamma$-strength function of the ${ }^{90} \mathrm{Y}$ compound nucleus, $M^{2}$ the normalization factor of the exciton model mean matrix element, and $a_{90}, a_{89}, a_{88}$, and $a_{87}$, the level density parameters of the ${ }^{90} \mathrm{Y},{ }^{89} \mathrm{Y},{ }^{88} \mathrm{Y},{ }^{87} \mathrm{Y}$ compound nuclei, respectively. Only 

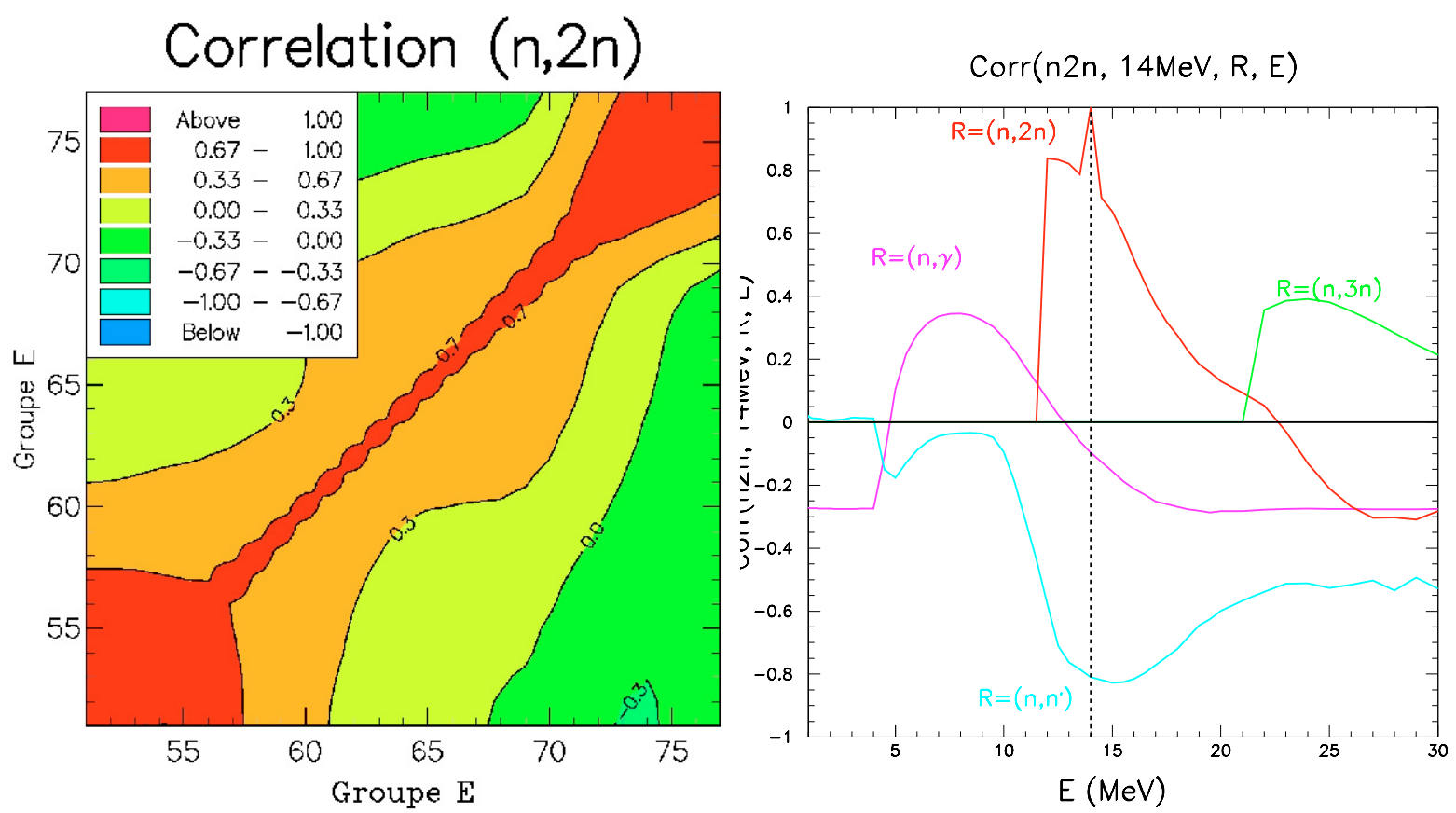

Fig. 4. In-channel correlatiosn matrix for the (n,2n) channel (left panel), and cross-channel slice of the full correlation matrix projected along the $14 \mathrm{MeV}(\mathrm{n}, 2 \mathrm{n})$ "axis" (right panel).

those six model parameters were introduced in the Backward Monte Carlo step since the covariance matrix of the four OMP parameters is already known. However, during the BFMC process, the four OMP parameters were sampled according to equations (6), (7) and (8). For the Backward Monte Carlo step, the data sets described in table 2 were used to build the generalized $\chi^{2}$ to be introduced into equation (2).

The $6 \times 6$ correlation matrix obtained after the Backward Monte Carlo step exhibits several features which are worth mentioning. The standard deviations are of the order of $12-15 \%$ for the level density parameters and $M^{2}$ preequilibrium parameter, and almost $20 \%$ for $\Gamma \gamma$. Moreover, the correlations are no longer small enough to be negligible. We get strong negative $(-0.78)$ correlation between $\Gamma \gamma$ and $a_{90}$, moderate positive (0.4-0.6) correlations between the level density parameters, and very weak correlations $(<0.1)$ of $M^{2}$ with other parameters. Given that $6 \times 6$ covariance matrix, its associated mean value vector, and the OMP $\pi_{1}(6)$ and $\pi_{2}(7,8)$ we build the $10 \times 10$ covariance matrix $\Pi_{2}$ for the TALYS parameters (with two uncorrelated $4 \times 4$ and $6 \times 6$ blocks) and its associated mean vector $\Pi_{1}$. These are then introduced in equation (1) to produce a sampling (of size $\mathrm{N}=5 \times 10^{5}$ samples) of the 10 considered model parameters. That sampling is then given as input to TALYS to produce a sampling of the calculated observables. The observables that were considered for the construction of the covariance matrix are the total $(\mathrm{n}, \gamma)$, total $\left(\mathrm{n}, \mathrm{n}^{\prime}\right)$, total $(\mathrm{n}, 2 \mathrm{n})$, total $(\mathrm{n}, 3 \mathrm{n})$, total $(\mathrm{n}, \mathrm{p})$, and total $(\mathrm{n}, \alpha)$ cross sections calculated on a 78 points energy mesh (between $1 \mathrm{keV}$ and $30 \mathrm{MeV}$ ). The covariance matrix obtained by analyzing the sampling of observables is a $468 \times 468$ matrix that includes both in-channel and cross-channel correlations.

Let us first discuss the diagonal components of that covariance matrix. Visually, the standard deviation bands plotted in figure 3 seem to account for the spread and error bars of the experimental data used in the BFMC process. The standard deviation associated with the $(\mathrm{n}, \gamma)$ cross section can be seen to vary between $30 \%$ and $50 \%$ over most of the considered energy range. The errors associated with the $\left(n, n^{\prime}\right)$ channel lie in the $8 \%$ to $12 \%$ range while the standard deviation of the $(n, 2 n)$ and $(n, 3 n)$ cross sections are both close to $5 \%$. These values seem to be larger than those given in the ENDF/B-VII files [5].

Examining the off-diagonal components of the covariance matrix is done by looking at slices of the corresponding correlation matrix. For example correlation between the cross sections for a given channel and the cross sections of the same channel at different energies can be plotted as in the left panel of figure 4. On this figure, which shows the "in-channel" correlation of the (n,2n) cross sections, strong positive correlations can be seen along the diagonal. However, for large energy differences (far off-diagonal) correlations can be negative, implying anti-correlations between the energies corresponding to opening of the $(n, 2 n)$ channel and those corresponding to the "closing of the $(n, 2 n)$ channel" (more precisely the beginning of competition with the $(n, 3 n)$ channel).

The above negative correlation and its probable link with the opening of another channel suggests that it might be worth looking at cross-channel correlations. The left panel of figure 4 shows such cross-channel correlations between the $(n, 2 n)$ cross section at $14 \mathrm{MeV}$ and the other channels and energies. On this graph we see positive and negative correlations occurring between different channels. A very simple explanation for the presence of negative correlations is linked to the unitarity of the models involved: once the reaction cross section has been determined by the optical potential, the sum of all the partial channels is fixed. Since 
that sum is fixed, if one channel increases, the sum of all the remaining channels must decrease, producing the negative cross-channel correlations that can be observed in figure 4 .

\section{Discussions}

By constraining the model parameter distribution by a generalized $\chi^{2}$ quality estimator, we construct a PDF of the model parameters that produce observables which are consistent with the experimental data. By sampling from that model parameter PDF, and calculating the associated observables, a sampling of the observable space is obtained which is analyzed in term of covariance matrix and mean value vector. That method was applied to the $n+{ }^{89} Y$ system modeled using the TALYS code with a semi-microscopic OMP. A full covariance matrix (including cross-channel correlations) for six channels and 78 energy points was produced and written in ENDF-6 [10] file 33 format.

We have shown that the BFMC method can be used as an alternative to the established Bayesian method for evaluating the uncertainties associated with nuclear data. Although covariances derived using these two methods are not exactly the same, they exhibit many similarities.

The BFMC-like and Bayesian approaches should be seen as complementary, having different strengths and weaknesses. Indeed, their respective fields of application do not completely overlap. For example, in cases where many model parameters are involved and too few experimental constraints exist, the Bayesian approach works well whereas the BFMC method faces too many unconstrained model parameters. One example of such a case is the determination the covariance matrix associated with isomeric ratios, which constitutes a very simple case for the Bayesian method (provided that some relevant experimental data exists), whereas the large number of parameters associated with the gamma cascade (and its continuum feeding) makes the BFMC method very unpractical.

Conversely, some cases benefit more from the BFMC approach. In order to evaluate the cross-section covariance matrix associated with the very sparsely measured $n+{ }^{88} Y$ system, the BFMC method benefits from parameter covariance matrices $\Pi_{2}$ and mean value vectors $\Pi_{1}$ that are already partly determined by a previous study of the well measured $n+{ }^{89} Y$ neighbouring system. More generally, the knowledge of the model parameter covariance matrix, which can be seen as a byproduct of the BFMC method, can profitably be reused in other studies. Insights into the role played by the various model parameters can also be gained by examining the parameter covariance matrix obtained by the BFMC method.

A possible evolution of the BFMC method will be to include integral experiments in the calculation of the $\chi^{2}$ along with the differential data that is presently used. That inclusion would introduce further constraints on the model parameter PDF that will presumably refine the final crosssection uncertainties, provided that integral and differential constraints are not contradictory.

Finally, since the calculation of the generalized $\chi^{2}$ that drives the Backward step relies on the knowledge the covariance matrix of experimental data, we urge experimentalists to provide complete covariance information with new measurements.

\section{References}

1. A.J. Koning, S. Hilaire, M. Duijvestijn, in Proceeding of the International Conference on Nuclear Data for Science and Technology, Santa Fe, 2004, edited by R.C. Haight, M.B. Chadwick, T. Kawano, P. Talou (AIP, New York, 2005), p. 1154; See also A.J. Koning (these proceedings).

2. M. Herman, P. Oblozinski, R. Capote, M. Sin, A. Trkov, A. Ventura, V. Zerkin, in Proceeding of the International Conference on Nuclear Data for Science and Technology, Santa Fe, 2004, edited by R.C. Haight, M.B. Chadwick, T. Kawano, P. Talou (AIP, New York, 2005), p. 1184.

3. C. Nordborg (these proceedings).

4. COVAR mailing list, http://www.nea.fr/lists/covar.html.

5. M.B Chadwick et al., Nucl. Data Sheets 107, 2931 (2006).

6. E. Bauge, J.P. Delaroche, M. Girod, Phys. Rev. C 63, 024607 (2001).

7. E. Bauge, M. Dupuis, in Proceedings of the Tours Symposium on Nuclea Physics V, Tours, France, 2003, edited by M. Arnoult, M. Lewitoviz, G. Münzenberg, H. Akimune, H. Utsunomiya, T. Wada, T. Yamagata (AIP, New York, 2004), p. 385.

8. E. Bauge, in Proceedings of the International Conference on Nuclear Data for Science and Technology, Santa Fe, 2004, edited by R.C. Haight, M.B. Chadwick, T. Kawano, P. Talou (AIP, New York, 2005), p. 1136.

9. E. Bauge, J.P. Delaroche, M. Girod, Phys. Rev. C 58, 1118 (1998).

10. Cross Section Evaluation working Group, ENDF-102 Data Format and procedures for the evaluated nuclear data file ENDF-6, edited by V. McLane, BNL-NCS-44945-01/04-Rev, 2001, Brookhaven National Laboratory. 